\title{
Human acellular amniotic membrane is adopted to treat venous ulcers
}

\author{
ZHOUPENG WU* ${ }^{*}$ XIAOYAN LIU*, DING YUAN and JICHUN ZHAO \\ Department of Vascular Surgery, West China Hospital, Sichuan University, \\ Chengdu, Sichuan 610041, P.R. China
}

Received December 20, 2017; Accepted April 26, 2018

DOI: $10.3892 /$ etm.2018.6331

\begin{abstract}
Venous lower limb ulcers are very common and affect $\sim 1 \%$ of the general population. The human acellular amniotic membrane (HAAM), which is isolated from the amniotic membrane (AM) via excluding the majority of cellular components, has lower antigenicity than the AM. The aim of the present study was to evaluate the effectiveness and safety of the HAAM, adopted to treat venous ulcers (VUs) of the lower extremities. The HAMM was isolated from the AM by the Stem Cells and Tissue Engineering laboratory at the West China Hospital of Sichuan University (Chengdu, China). HAMMs were grafted onto VUs in 4 patients, with follow-up evaluations performed on the 3rd day and at the end of the 1st, 2nd and 3rd week, and 2nd, 3rd and 6th month after the HAAMs were applied. The size and depth of the VU (determined based on whether the depth of VU reaches the tibial plane), the proportion of granulation tissue (whether $>50 \%$ ) and the degree of secretion (measured by asessing the degree of satuation in the outer gauze) and infection (assessed qualitatively via the appearance of purulence or peripheral swelling) were assessed. Pain score was monitored at the same intervals using a visual analog scale. Complete epithelialization (healed tissue) occurred in 2 cases: The first at the end of the 3 rd week and the second, at the 2nd month following HAAM induction. In one of the remaining cases, ulcer size was reduced by $>60 \%$; however the ulcer size of the remaining case only reduced by $<20 \%$. Overall, the size of ulcer in cases $1,2,3$ and 4 decreased to $1.2 \times 1.1$ and $1.4 \times 0.4 \mathrm{~cm}, 1.3 \times 1.8$ and $2.3 \times 1.4$, respectively, with evident decreases in ulcer depth. The proportion of granulation tissue in each case was $>50 \%$. Furthermore, purulence and secretion completely disappeared in all 4 cases. Additionally, the medical cost of HAAM treatment is
\end{abstract}

Correspondence to: Dr Ding Yuan, Department of Vascular Surgery, West China Hospital, Sichuan University, 37 GuoXue Alley, Chengdu, Sichuan 610041, P.R. China

E-mail: liuyonghhz@163.com

${ }^{*}$ Contributed equally

Key words: human acellular amniotic membrane, venous ulcer substantially lower than that of AM treatment, skin autografts and biomaterial transplantation, thus alleviating the patients' financial burden. These findings suggest that HAMM was highly effective in treating VUs in patients.

\section{Introduction}

Venous ulcers (VUs) are ulcerative changes caused by chronic venous insufficiency. These types of ulcers have a high recurrence rate with no simple treatment determined for an effective short-term outcome. This severely influences the patients' life quality and causes a heavy burden following a long treatment period (1). Therapy plans for VUs typically include drugs, skin transplantation and surgery (2). Drug therapy works slowly, whereas the therapeutic effect is frequently not obvious, though it is defined as a basic treatment for VUs (2). Skin transplantation provides a promising outcome within a short period, yet severe surgical trauma is inevitable (2). Furthermore, dermal biomaterial are expensive and often unaffordable for patients. The amniotic membrane (AM) has advantages of epithelialization stimulation, anti-bacterial properties and definite mechanical strength. Consequently, the use of the AM has a well-established history in healing burn wounds and corneal ulcers (2). Cases of adopting the AM to heal VUs have also been reported worldwide due to its beneficial attributes (2-4). However, the AM is not convenient to store, as it requires a temperature of $-80^{\circ} \mathrm{C}$ and a sterile vial $(3,4)$. Hence, expensive medical costs are incurred by the patients. In contrast, the human acellular amniotic membrane (HAAM) has overcome this disadvantage. HAAM is the natural extracellular matrix of the human amnion membrane, which contains fibronectin, laminin, elastin, proteoglycans, hyaluronan, collagens I, III, IV, V, and VII, basic-fibroblast growth factor, epidermal growth factor and transforming growth factor- $\beta$, with multiple bioactive factors $(2,4)$. HAAMs retain effective components and exclude the majority of cells, which results in weak antigenicity (2). Additionally, HAAM was demonstrated to be anti-inflammatory, anti-microbial and non-tumorigenic, producing few ethical issues (3). Furthermore, the remaining basal membrane layer and strata compactum are easy to store, transport and use (2). Furthermore, theoretically, the HAAM is safer than the AM, yet it is equally effective (2-4). All these parameters have made HAAM a cyto-compatible membrane with various bioactive factors, which is widely applied in 
tissue engineering and within clinics. The HAAM is isolated from the AM via cleansing the cellular components (4). Thus, a pilot study regarding the efficiency and safety of HAAM adopted for the treatment of VUs was conducted.

\section{Patients and methods}

Clinical data. A total of 4 patients, who received HAAM therapies in West China Hospital (Chengdu, China) from January-April 2013 and were diagnosed with VU of the lower limbs were included in the present pilot study. The patients' left extremities had swollen to varying degrees. All ulcers had medium-considerable purulence and considerable secretion. The area surrounding the ulcers appeared painful. Furthermore, long-term drug therapy had been ineffective in the 4 patients prior to admission. Following admission, all patients underwent an ultrasound examination. Consequently, 2 patients were diagnosed with left lower extremity varicose veins (stage C6) and the remaining 2 patients were diagnosed with post-thrombotic syndrome (PTS; stage 4b). All 4 patients, who received HAAM treatment, were followed up for $\sim 6$ months. The study protocol conformed to the ethical guidelines of the 1975 Declaration of Helsinki, with approval granted by the Human Research Review Committee at West China Hospital, Sichuan University. All patients provided written informed consent.

Preparation of HAAM. HAAM were prepared by chemical detergent-enzymatic extraction at the Stem Cells and Tissue Engineering Laboratory of the West China Hospital of Sichuan University. Fresh human amnion was cross-linked with glutaraldehyde, then shaken in $0.5 \%$ SDS for $24 \mathrm{~h}$ at $4^{\circ} \mathrm{C}$, and finally treated with $0.25 \%$ trypsin for $4 \mathrm{~h}$ at $37^{\circ} \mathrm{C}$. The product was freeze-dried and sterilized using ethylene oxide. Human fibroblasts were isolated from embryos, expanded in vitro using an amplification kit (Turely Cell VGA kit performed according to the manufacturer's protocol; (Sichuan Neo-Life Stem Cell Biotech, Inc., Sichuan, China) for $48 \mathrm{~h}$ at $37^{\circ} \mathrm{C}$ and seeded in HAAM. The HAAMs were stained for $4 \mathrm{~h}$ at $4^{\circ} \mathrm{C}$ with hematoxylin and eosin and were examined under light microscopy (magnification, x400). Then using Mallory's stain, samples were fixed in $3 \%$ glutaraldehyde with $0.1 \mathrm{M}$ phosphate buffer ( $\mathrm{pH} 7.2$ ) for $120 \mathrm{~min}$ at $4^{\circ} \mathrm{C}$. Samples were post-fixed in $1 \%$ osmium tetroxide for $60 \mathrm{~min}$ at room temperature, and dehydrated in 50, 70, 80, 90, and $100 \%$ ethanol for $10 \mathrm{~min}$. The samples were then air-dried, mounted, sputter coated with gold and visualized using a Hitachi S-4800 scanning electron microscope (magnification, x500; Hitachi, Ltd., Tokyo, Japan). It was confirmed that there were no cell residues in the HAAM. One side of the HAAM had a reticular and porous structure, whereas the other side had a compact, fibrous structure. The pore diameter ranged from $10-80 \mathrm{~nm}$.

Protocol for use of HAAM. Patients initially received drug therapy and symptomatic treatment, including $500 \mathrm{mg}$ Alvenor (Servier Laboratories, Nueilly-sur-Seine, France) twice daily with elevation of the affected limb. Subsequently, the VU was disinfected with povidone-iodine solution prior to the application of HAAM, in order to ensure the VU did not become infected.
Once no purulence or secretion exuded from the VU was observed, a suitably sized HAAM (Chengdu Qingshan Likang Pharmaceutical Co., Ltd., Chengdu, China) was used to completely cover the VU (the first layer). An asepsis gauze saturated with heparinized water $(100 \mathrm{mg} / 500 \mathrm{ml})$ was placed on the HAAM (the second layer), then covered with an oiled gauze (the third layer) and, finally, another asepsis gauze was deposited on the oiled gauze (the fourth layer). The dressing materials were replaced (except the first layer) every 2 days. No iodine solution or ethyl was used to disinfect the inner layer, which was soaked with normal saline instead. Furthermore, no scraping was performed. Replacement of the outer dressing materials was repeated every 2 days for the initial 14 days.

Evaluation index for ulcer treatment. The evaluations were carried out on the 3rd day and at the end of the 1st, 2nd and 3rd week, and the 2nd and 3rd month after the HAAMs were adopted. The size and depth of the VU (determined based on whether the depth of VU reaches the tibial plane), the proportion of granulation tissue (whether $>50 \%$ ) and the degree of secretion (measured by asessing the degree of satuation in the outer gauze) and infection (assessed qualitatively via the appearance of purulence or peripheral swelling) were evaluated. If the size and depth of the ulcer increased during therapy, the HAAM treatment was considered to have failed, whereas if the size and depth of the ulcer did not change, the HAAM treatment was deemed null. Pain scores were also assessed at the same intervals using a visual analog scale (4-6), where 0 was no pain and 10 was the worst pain imaginable. Patients chose a suitable score to represent and quantify the pain they suffered on admission and during therapy. Secretion evaluation was measured by the saturation degree of the outer gauze. Infection was qualitatively assessed via the appearance of purulence or peripheral swelling (4).

\section{Results}

Patient characteristics. As presented in Table I, the 4 included patients were all male, with a median age of $62.3 \pm 2.2$ years. All received drug therapy. A total of 2 patients were diagnosed with left lower extremity varicose veins coupled with VU, whereas the remaining 2 patients exhibited PTS coupled with VU. The sizes of VU ranged from $3.2 \times 0.9$ to $5.1 \times 2.5 \mathrm{~cm}^{2}$, which are presented in Table I. One VU accessed the tibia and the other three were confined to the tissue.

Evaluation of HAAM treatment effect. On the 3rd day after HAAM was adopted to treat the VUs, the HAAM had firmly adhered to the surface of the VU. A condition evaluation revealed $100 \%$ adherence rate, ensuring long-term HAAM efficacy. Complete epithelialization (healed tissue) occurred in 2 cases (case 1 and 2): The first at the end of the 3rd week and the second, at the 2nd month following HAAM induction. In case 3 , ulcer size was reduced by $>60 \%$; however, the ulcer size of the remaining case (case 4 ) only reduced by $<20 \%$. Overall, the mean size of ulcer reduction in each case was $>50 \%$, case 1 and 2 decreased to $1.2 \times 1.1$ and $1.4 \times 0.4 \mathrm{~cm}$, and case 3 and 4 decreased to $1.3 \times 1.8$ and $2.3 \times 1.4$, respectively, with evident decreases in ulcer depth at the end of $3 \mathrm{rd}$ week. The proportion of granulation tissue in each case was $>50 \%$. Nearly all ulcers 
Table I. Baseline characteristics of included patients.

\begin{tabular}{|c|c|c|c|c|c|c|}
\hline Patient & Gender & $\begin{array}{l}\text { Age } \\
\text { (years) }\end{array}$ & $\begin{array}{l}\text { Size of VU } \\
(\mathrm{cm} \times \mathrm{cm})\end{array}$ & Depth of VU & History & Diagnosis \\
\hline Case 1 & Male & 60 & $3.4 \times 2.7$ & No access to tibia & $\begin{array}{l}\text { Appeared for }>6 \text { months } \\
\text { and not healed }\end{array}$ & $\begin{array}{l}\text { Left lower extremity varicose } \\
\text { vein coupled with VU }\end{array}$ \\
\hline Case 2 & Male & 61 & $2.8 \times 2.3$ & No access to tibia & $\begin{array}{l}\text { Appeared for }>4 \text { months } \\
\text { and not healed }\end{array}$ & PTS coupled with VU \\
\hline Case 3 & Male & 63 & $5.1 \times 2.5$ & No access to tibia & $\begin{array}{l}\text { Appeared for }>5 \text { months } \\
\text { and not healed }\end{array}$ & $\begin{array}{l}\text { Left lower extremity varicose } \\
\text { vein coupled with VU }\end{array}$ \\
\hline Case 4 & Male & 65 & $3.2 \times 0.9$ & Access to tibia & $\begin{array}{l}\text { Appeared for }>12 \text { months } \\
\text { and not healed }\end{array}$ & PTS coupled with VU \\
\hline
\end{tabular}

All of the ulcers were located on the surface of the pretibial skin. VU, venous ulcer; PTS, post-thrombotic syndrome.

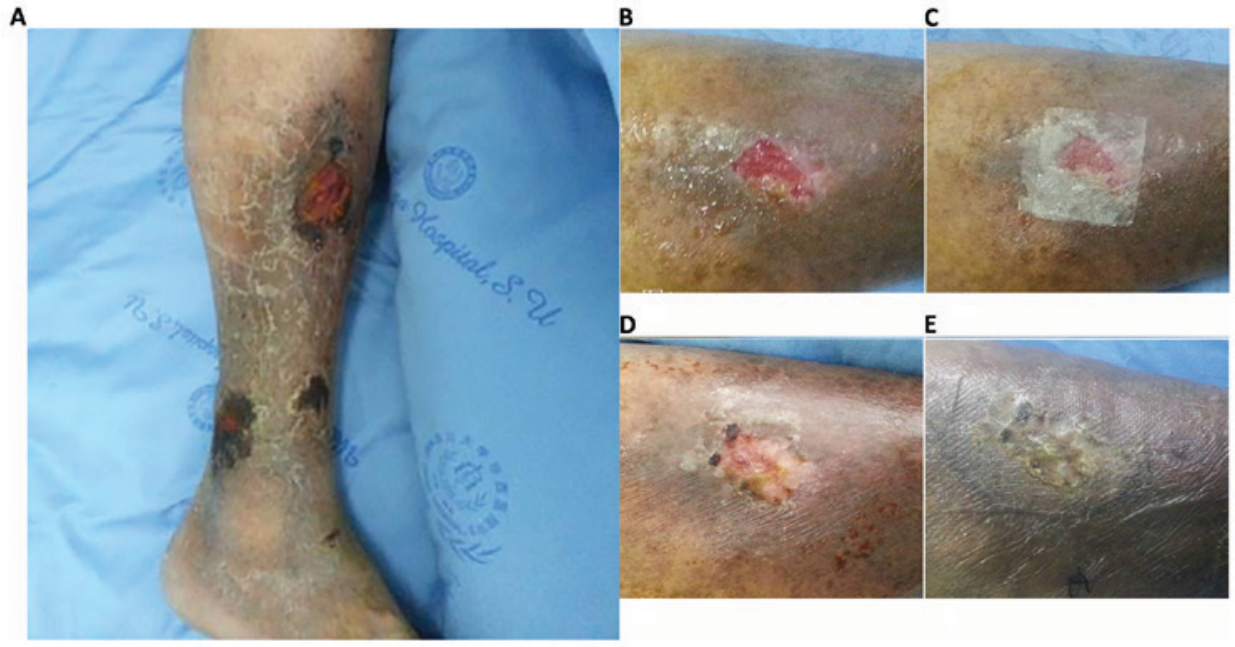

Figure 1. Size change during HAMM treatment period in one patient during follow-up. (A) VU prior to treatment. (B) VU following preparation. (C) VU with HAAM coverage. (D) VU at 1 week post-treatment. (E) VU at 2 weeks post-treatment. HAMM, human acellular amniotic membrane; VU, venous ulcer.

had decreased in size and/or depth at the end of the 1st week after the HAAM was applied, except for case 4. The onset of the HAAM effect was apparent within a shorter time for the smaller VUs compared with the larger ones. Consequently, the therapeutic effect, measured by size and depth change per day, was observed earlier for the smaller VUs, whereas relatively larger sized VUs improved slowly. The detailed changes in all four patients are presented in Figs. 1 and 2.

Symptoms associated with VUs. On the 3rd day following HAAM therapy, no secretion was observed in 3 cases. Some exudation occurred in 1 case but there was no purulence and at 1 week of therapy, this ulcer became dry, with no exudation evident. A total of 2 patients (case 1 and 4) did not feel pain surrounding the ulcer during HAAM therapy. Also, the pain was relieved in the remaining 2 patients (case 2 and 3), with the pain scores reduced by 3 and 2 points to 1 and 2 point(s), respectively, at 1-week of therapy, followed by 0 pain in both patients. Detailed changes in all the cases are presented in Fig. 3.

Safety and convenience of HAAM. No uncomfortable symptoms occurred during the HAAM therapy. No local or general

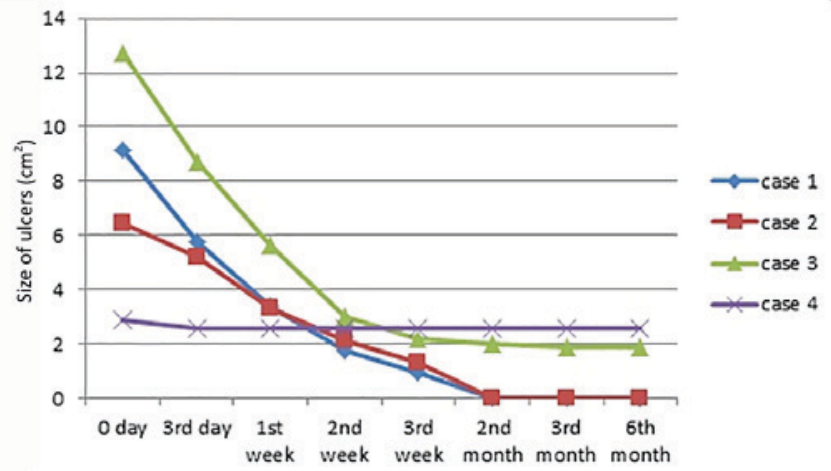

Figure 2. Size change during human acellular amniotic membrane treatment period and follow-up. Size was measured in $\mathrm{cm}^{2}$. In case 4 , although at the 6-month follow-up there was no marked change in size, there was no purulence or secretion.

inflammation or allergic reactions were noted. In addition, the HAAM therapy protocol was simple to manage: 2 patients (case 3 and 4) dressed the wound by themselves following discharge. 


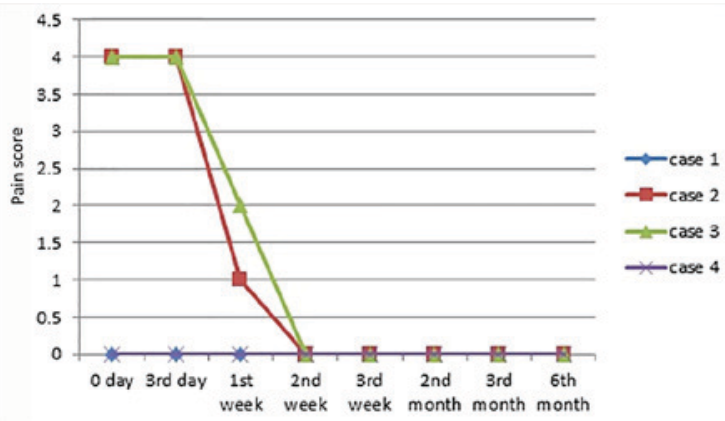

Figure 3. Pain score change during HAAM treatment. A total of 2 patients (cases 1 and 4) did not report pain around the ulcer during HAAM therapy. Furthermore, the pain was relieved in the remaining 2 patients (cases 2 and 3), with the pain score reduced by 3 and 2 points to 1 and 2 point(s), respectively, at 1-week of therapy, and subsequently, was reported as 0 in both patients. HAMM, human acellular amniotic membrane.

Medical cost of HAAM. The mean medical cost of AM graft treatment is estimated at $¥ 8,256$, whereas HAAM treatment cost for each patient at the West China Hospital is $¥ 5,276$ (7). In comparison, the mean cost of an autograft and biomaterial substitute is $¥ 12,435$ and $¥ 23,643$, respectively (7). Thus, HAAM's cost-efficiency is markedly high. A detailed comparison of the mean costs of these treatments is presented in Fig. 4.

\section{Discussion}

The efficacy of the AM, to treat various kinds of ulcers, has been previously confirmed (5), yet the application of the $\mathrm{AM}$ to treat VUs of the lower limbs has only emerged more recently $(4,6)$. In addition, although AM is an alloplastic biomaterial, any untoward reaction of the AM is rarely reported. Hence, considering its safety and efficiency, the use of AMs to treat VUs is clinically recommended $(2,6)$. In the present study, the safety and efficacy of HAAM was explored. Furthermore, to the best of our knowledge, the present study is the first to detail practice experience regarding HAAM applied in VU treatment. In the present study, it was evident that the size and/or depth of most ulcers improved at 1-week following HAAM treatment, irrespective of the VU size. Particularly, when the ulcer was relatively small, the therapeutic effect of HAAM is more impressive; namely, the ulcers healed completely (epithelia appeared and granulation tissue formed) between 1 and 2-week post-treatment. Despite this effect, we found the HAAM is mostly effective in the first two weeks, which was confirmed by the previous study by Mermet et al (4). The present data indicated that the decrease in ulcer size range for the 3 cases (excluding case 4) was more intense in the first 2 weeks than during the subsequent duration. The total size decrease of the four ulcers was estimated as $21.77 \mathrm{~cm}^{2}$ in the first 2 weeks, which is substantially greater than $4.99 \mathrm{~cm}^{2}$, which was recorded in the final 2 weeks of therapy. It was demonstrated that the depth of the wound may affect the healing speed; the deeper the wound, the longer time it takes to heal. The size reduction of three ulcers (cases 1-3) was evident, with all decreasing by $>80 \%$ (85-100\%). In contrast, in case 4 , the ulcer size decreased by $<10 \%$. However, its depth and secretion reduced dramatically. It has been reported that HAAM is particularly effective in

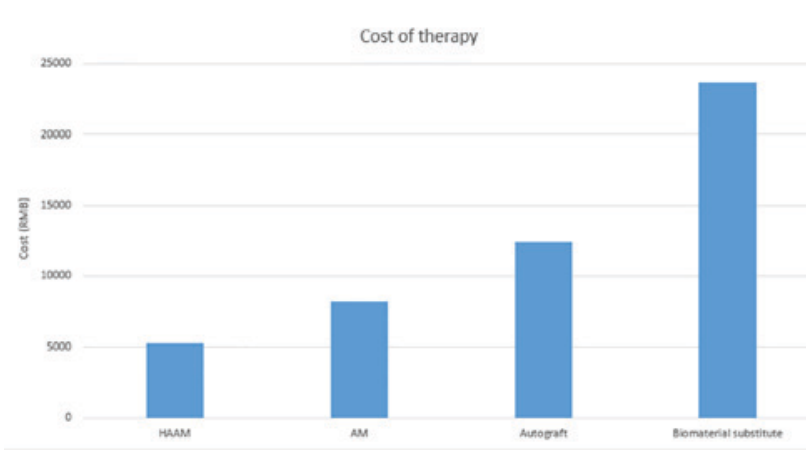

Figure 4. Comparison of medical costs between particular methods used to treat venous ulcers. All data represent the cost at the West China Hospital (Chengdu, China). The medical cost refers only to the biomaterial fee and does not include all costs (rate of exchange between Chinese RMB and US dollar is 6.2:1). HAMM, human acellular amniotic membrane; AM, amniotic membrane.

shrinking shallow ulcers, rather than deep ulcers (6). This was also reported in Sawhney's research on the AM as a biological dressing in the management of burns (8). The mean healing time was reported to be significantly faster in all groups with amnion coverage than in controls (superficial, 9.3 vs. 12.5 days; intermediate, 15.7 vs. 23.9 days; and deep, 27.5 vs. 37.5 days). The authors concluded that as the depth of the wound increased, healing time increased, although this needs to be validated in a future study. The current study provided conclusive evidence that HAAM therapy was able to shrink the VUs, while simultaneously maintaining the ulcers free from secretion and purulence.

The underlying pathophysiology of unhealed VU is repeated inflammatory reaction. Fortunately, anti-inflammation and anti-bacterial properties are main characteristics of HAAM $(9,10)$. Owing to the existence of T lymph cells, lysozymes, and thrombin, the HAAM has a powerful anti-bacterial property and ability to absorb wound secretion $(11,12)$. Although numerous cellular components are excluded from the AM in preparing the HAMM, the therapeutic effect of the HAAM is not affected (11). During HAAM therapy, the pain score was lower than that of dressing therapy (12), which may help to ensure patients' compliance. The possible reasons for the low pain score can be attributed to various factors. First, the key premise of successful HAAM treatment and lower pain score rely on the prior preparation of the ulcers, which would create an initial optimal environment for HAAM. Second, the absence of scraping assists to preserve the integrity of the HAAM, which is essential for its efficacy. Third, the anti-inflammation function of the HAMM, which decreases and weakens the stimulation of local nerve endings, may be another crucial reason contributing to the low pain scores recorded.

Compared with the AM, weak antigenicity is the major advantage of the HAAM (7). In the present study, no local or general allergic reaction occurred in any of the evaluated cases. Removing serum human leukocyte antigen (HLA)-I from the HAAM is paramount (13), to avoid allergic reactions and ensure safety. Convenience and low cost are additional benefits of the HAAM compared with the AM. The AM is fragile, requiring particular storage conditions, thus AM appliance incurs a relatively higher cost than the 
HAAM $(9,10)$. An AM graft is estimated to cost $¥ 2,122$ (AM therapy typically requires needs $2-3$ pieces of AM; the mean medical fee is $¥ 8,256$, ranging from $¥ 7,580-10,500$ ), whereas the HAAM costs patients $<¥ 1,000$ (HAAM therapy requires 1-3 pieces of HAAM; the mean medical fee is $¥ 5,276$, ranging from $¥ 4,700-6,700$ ), at the West China Hospital. Also, compared with the mean cost of an autograft $(¥ 12,435)$ and biomaterial substitute ( $¥ 23,643$ ), the HAAM has high cost-efficiency. A similar cost trend is observed in Spain (6). However, the main limitation of the present study is the lack of serum HLA-I detection in the patients. The HAAM is acquired by removing the majority of the cells from the $\mathrm{AM}$, therefore, a loss of growth factors and precursor cells is inevitable, which may have affected the results. Therefore, a direct comparison between the HAAM and AM is not feasible, so it cannot be concluded whether HAAM is more effective than the AM. Nevertheless, in the present study, no treatment fails were recorded in all four cases evaluated. Furthermore, the effect is similar to the AM in terms of the wound healing $(9,10)$. Accordingly, the present findings suggest that HAAM has a promising effect.

The present study explored the therapeutic effect of the HAAM, adopted to treat VUs via analyzing a number of cases. Although the present study cannot prove whether HAAM has a determined effect on VUs, the HAAM displayed an impressive tendency for the treatment of VUs, providing a short therapy duration, low medical cost and simple dressing procedure. However, regardless of the HAAM efficacy, drug therapy and compression stockings remain the fundamental methods for VU treatment. Therapy for VU also includes surgery, skin autografts and biomaterial transplantation. The HAAM is an innovative way to clinically treat VUs and provides epithelial stimulation, pain relief and a simple dressing procedure, as well as lower cost compared with the AM, autograft or biomaterial transplantation. Thus, future studies on adopting HAMMs to treat ulcers is worthwhile for physicians and researchers.

\section{Acknowledgements}

Not applicable.

\section{Funding}

The presnt study was supported by the Technology Research and Development Program of Sichuan Province, China (grant. no. 2016SZ0060).

\section{Availability of data and materials}

The datasets used and/or analyzed during the current study are available from the corresponding author on reasonable request.

\section{Authors' contributions}

DY designed the present study; ZW and XL wrote the manuscript and collected data; DY and JZ supervised experiements to ensure they were conducted correctly and revised the manuscript. JZ also contributed to the conception and design of the study, manuscript revision and final approval of the version to be published.

\section{Ethics approval and consent to participate}

The study protocol conformed to the ethical guidelines of the 1975 Declaration of Helsinki, with approval granted by the Human Research Review Committee at West China Hospital, Sichuan University (Chengdu, China). All patients provided written informed consent.

\section{Patient consent for publication}

All patients provided written informed consent.

\section{Competing interests}

The authors declare that they have no competing interests.

\section{References}

1. Patel NP, Labropoulos N and Pappas PJ: Current management of venous ulceration. Plast Reconstr Surg 117 (Suppl): S254-S260, 2006.

2. Lo V and Pope E: Amniotic membrane use in dermatology. Int J Dermatol 48: 935-940, 2009.

3. Litwiniuk M, Bikowska B, Niderla-Bielińska J, Jóźwiak J, Kamiński A, Skopiński P and Grzela T: Potential role of metalloproteinase inhibitors from radiation sterilized amnion dressings in the healing of venous leg ulcers. Mol Med Rep 6: 723-728, 2012.

4. Mermet I, Pottier N, Sainthillier JM, Malugani C, Cairey-Remonnay S, Maddens S, Riethmuller D, Tiberghien P, Humbert P and Aubin F: Use of amniotic membrane transplantation in the treatment of venous leg ulcers. Wound Repair Regen 15: 459-464, 2007.

5. Singh R, Chouhan US, Purohit S, Gupta P, Kumar P, Kumar A, Chacharkar MP, Kachhawa D and Ghiya BC: Radiation processed amniotic membranes in the treatment of non-healing ulcers of different etiologies. Cell Tissue Bank 5: 129-134, 2004.

6. Gutiérrez-Moreno S, Alsina-Gibert M, Sampietro-Colom L, Pedregosa-Fauste S and Ayala-Blanco P: Cost-benefit analysis of amniotic membrane transplantation for venous ulcers of the legs that are refractory to conventional treatment. Actas Dermosifiliogr 102: 284-288, 2011.

7. Luo JC, Li XQ and Yang ZM: Preparation of human acellular amniotic membrane and its cytocompatibility and biocompatibility. Zhongguo Xiu Fu Chong Jian Wai Ke Za Zhi 18: 108-111, 2004.

8. Sawhney CP: Amniotic membrane as a biological dressing in the management of burns. Burns 15: 339-342, 1989.

9. Beele H, de la Brassine M, Lambert J, Suys E, De Cuyper C, Decroix J, Boyden B, Tobback L, Hulstaert F, De Schepper S, et al: A prospective multicenter study of the efficacy and tolerability of cryopreserved allogenic human keratinocytes to treat venous leg ulcers. Int J Low Extrem Wounds 4: 225-233, 2005.

10. Bailo M, Soncini M, Vertua E, Signoroni PB, Sanzone S, Lombardi G, Arienti D, Calamani F, Zatti D, Paul P, et al: Engraftment potential of human amnion and chorion cells derived from term placenta. Transplantation 78: 1439-1448, 2004.

11. Walker AB, Cooney DR and Allen JE: Use of fresh amnion as a burn dressing. J Pediatr Surg 12: 391-395, 1977.

12. Ganatra MA: Amniotic membrane in surgery. J Pak Med Assoc 53: 29-32, 2003.

13. Kubo M, Sonoda Y, Muramatsu R and Usui M: Immunogenicity of human amniotic membrane in experimental xenotransplantation. Invest Ophthalmol Vis Sci 42: 1539-1546, 2001.

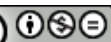

This work is licensed under a Creative Commons Attribution-NonCommercial-NoDerivatives 4.0 International (CC BY-NC-ND 4.0) License. 